\title{
Preparation and Sensing Properties of Organic Gel Fluorescence Films Based on ZnS Nanoparticles
}

\author{
XIA Huiyun *, GENG Tong, ZHAO Xu, LI Fangfang, WANG Fengyan, GAO Lining \\ School of Materials Science and Engineering, Chang'an University, Xi'an 710062, P. R. China.
}

\begin{abstract}
Accurate and rapid detection of organic amines in the vapor phase is essential for various applications such as agricultural use, industrial and environmental testing, and food security. Supramolecular gels composed of cholesterol derivative-based low-molecular-mass gelators (LMMGs) have attracted considerable attention owing to their unique character and formation mechanisms. In this study, a ZnS-supramolecular organogel hybrid film for amine vapor sensors was reported. It must be pointed out that the method of preparation of hybrid films considered

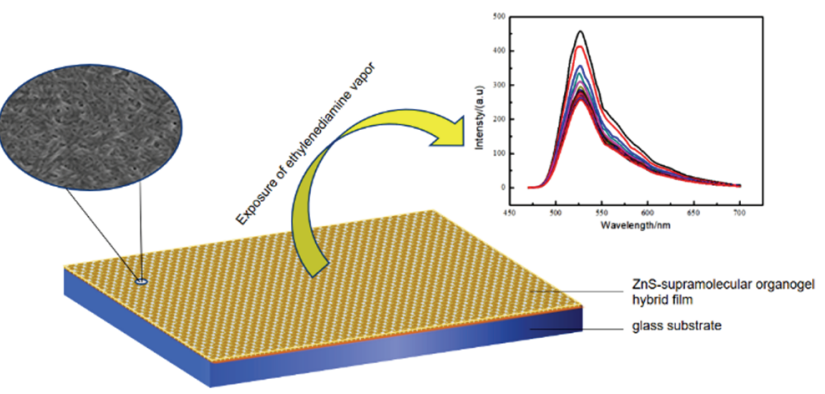
here is different from that of the ZnS-organogel hybrid films previously reported. Because the sensing performance of nanomaterials strongly depends on their nanostructures, it is expected that nanomaterials synthesized by different methods exhibit different nanostructures and ultimately different sensing properties. The luminescent $\mathrm{ZnS}$ nanoparticles were first prepared by the oil-water interface method, before being dispersed in an organic solution containing the LMMG. Finally, the aforementioned solution was casted onto the surface of a glass substrate to fabricate a $\mathrm{ZnS}$-supramolecular organogel fluorescent hybrid film after drying at room temperature. Scanning electron microscopy observations revealed that the surface morphology of the hybrid film was uniform cross-linked nanofibers. Transmission electron microscopy results revealed that the average particle size of the obtained $\mathrm{ZnS}$ nanoparticles is about $200 \mathrm{~nm}$. The crystal structure of the $\mathrm{ZnS}$ nanoparticles is cubic, as revealed by X-ray diffraction. The photoluminescence emission spectra of the ZnSsupramolecular organogel film were recorded for various quantities of $\mathrm{ZnS}$ loading; the maximum emission wavelength of the hybrid films hardly changed, indicating that the dispersity of the $\mathrm{ZnS}$ nanoparticles in the hybrids is very well. Because the film network formed by the gelator has a good confinement effect on the ZnS nanoparticles, the hybrid film exhibits stable luminescence performance. Sensing experiments showed that the hybrid films are sensitive to the existence of organic monoamine and diamine vapors, and the sensitivity improved as the dosage of $\mathrm{ZnS}$ nanoparticles was increased. The quenching mechanism was discussed by comparing the fluorescence lifetimes of the hybrid films in the presence of air and ethylenediamine (EDA) vapor. It was found that the sensing mechanism is mainly static quenching, with a very small amount of dynamic quenching. The sensing performances of the film for common volatile organic compounds were investigated with a detection limit of $10.13 \mathrm{ppm}\left(1 \mathrm{ppm}=1 \times 10^{-6}\right.$, volume fraction) obtained for the EDA vapor. Reversible experiments indicated that the films have a good reversible response in the presence of EDA vapor. It is anticipated that this type of supramolecular organogel hybrid film could find applications in the monitoring of volatile organic amines in the areas of industry and environment.
\end{abstract}

Key Words: ZnS nanoparticles; Supramolecular organic gels; Fluorescence; Hybrid film; Sensing properties

Received: January 31, 2018; Revised: March 2, 2018; Accepted: March 2, 2018; Published online: March 8, 2018.

*Corresponding author. Email: xiahy@chd.edu.cn; Tel.: +86-29-82337340.

The project was supported by the National Natural Science Foundation of China (51202016, 51502021), Natural Science Basic Research Plan in Shaanxi Province, China (2017JQ2025, 2017JQ2003), Xi'an Science and Technology Planning Project, China (2017137SF/WM031).

国家自然科学基金(51202016, 51502021), 陕西省自然科学基础研究计划(2017JQ2025, 2017JQ2003)及西安市科技厅计划项目(2017137SF/WM031)资 助

(C) Editorial office of Acta Physico-Chimica Sinica 


\title{
基于 ZnS 纳米粒子的有机凝胶苂光薄膜的制备及其传感性能
}

\author{
夏慧芸 ${ }^{*}$, 耿通, 赵旭, 李芳芳, 王风燕, 高莉宁 \\ 长安大学材料科学与工程学院, 西安 710062
}

\begin{abstract}
摘要: 本文首先采用油水界面法制备发光纳米ZnS粒子, 再通过物理混合法, 将其分散在溶有小分子胶凝剂的有机溶液 中, 流延于玻璃基质表面, 得到ZnS苂光薄膜。实验结果表明, ZnS纳米粒子的平均粒径大小约为 $200 \mathrm{~nm}$, 具有立方晶 型结构, 并且在杂化薄膜中具有良好的分散性; 胶凝剂形成的网络结构对ZnS纳米粒子具有良好的限域效应, 表现为稳 定的发光性能; 气敏实验表明, 该杂化膜对挥发性有机单胺和二胺具有灵敏的选择性传感作用; 且其灵敏度随着杂化薄 膜中ZnS担载量的增大逐渐提高; 可逆性实验表明该薄膜对乙二胺蒸汽具有良好的可逆响应性。
\end{abstract}

关键词: ZnS纳米粒子; 超分子有机凝胶; 荧光; 杂化薄膜; 传感性能 中图分类号: 0648

\section{1 引言}

近年来, 随着科技不断发展, 环境污染产生的 问题日益突出, 对环境造成污染的有害物质的质 量监测研究也越来越受到人们的关注。质量检测 主要是检测有害物质的种类及其浓度。作为农业 肥料、医学药品、生物缓冲剂和填充颜料的重要原 料之一的胺类物质 ${ }^{1-3}$, 由于其毒性和挥发性非常 强, 甚至能够致癌, 已经成为自然环境中常见的污 染物, 其高灵敏检测技术的开发迫在眉睫。据文献 报道 ${ }^{4}$, 用于液相胺类化合物检测的工作较多, 但 是气相较少, 因此发展对气相胺类物质选择性佳、 稳定性好、使用便捷的传感材料或者分析方法尤 为重要。

用于胺类化合物检测的方法包括气液相色谱 法、质谱法、苂光法和电化学法等 ${ }^{5-7}$ 。色谱、质 谱法虽然灵敏度高, 但是仪器价格比较贵, 仪器携 带不方便, 很难进行实时实地检测, 并且测试前样 品预处理过程繁琐复杂, 严重影响了该方法的推 广。苂光分析法具有操作简单、灵敏度高以及选择 性好等优点, 已广泛成功用于多种化学物质的检 测 ${ }^{8-11}$, 加之薄膜传感器对待测体系不产生污染, 可重复使用, 易于器件化等优点, 使得苂光传感薄 膜的设计及制备倍受广大科研工作者的青睐 ${ }^{12}$ 。

大量小分子有机胶凝剂(LMOG)在分子间弱 相互作用力的作用下能够自组装形成具有三维网 络结构的超分子凝胶, 其中胶凝剂孔道间固有的 毛细作用能够使溶剂分子的流动受阻, 最终使体 系胶凝化 ${ }^{13-15}$ 。该三维网络结构是无机纳米粒子 成核及其生长的良好载体, 可有效避免无机纳米 粒子的聚集, 大大提高其稳定性。事实上以有机凝 胶为模板, 人工模拟生物矿化作用, 采用原位生成 法已经制备得到多种形貌各异、传感性能优异的
半导体-超分子凝胶杂化薄膜材料 ${ }^{16-18}$ 。但是以超 分子凝胶为基质制备无机纳米结构的传感较少, 而且关于硫化物传感性能的研究主要是通过掺杂 无机物进行改性提高, 故针对单独使用硫化物半 导体的传感研究仍需进一步深入 ${ }^{19-22}$ 。

事实上, 我们课题组之前针对 $\mathrm{ZnS}$-超分子凝 胶杂化薄膜的制备及传感性能就有所研究 ${ }^{11}$, 与 本工作区别在于传感元素 $\mathrm{ZnS}$ 纳米粒子的制备方 法不同, 前者为原位沉积法, 该工作采用油水界面 法制备 $\mathrm{ZnS}$ 纳米粒子, 再将其分散于溶有小分子 胶凝剂的有机溶液中, 流延法得到 $\mathrm{ZnS}$ 苂光薄膜。 由于纳米材料的传感性能强烈依赖于其纳米结 构, 因此预期不同制备方法制得的纳米材料必将 会得到不同的纳米结构, 最终表现为不同的传感 性能。故本文通过物理共混法, 以超分子凝胶薄膜 作为载体, 将新鲜制备的 $\mathrm{ZnS}$ 纳米粒子担载于薄 膜内部, 采用 $X$ 射线衍射 $(X R D) 、$ 扫描电子显微 镜 (SEM)、紫外可见近红外分光光度计 (UV-Vis$\mathrm{NIR}$ )、光致发光光谱(PL)和寿命 (Lifetime)等手段 对 $\mathrm{ZnS}$ 纳米粒子的结构、薄膜表面结构、发光性 能、传感性能及传感机理进行系统研究。

\section{2 实验部分}

\section{1 试剂与仪器}

硬脂酸锌购自天津市福晨化工厂, 硫化钠、油 酸和环己烷均购自天津市天力化学试剂有限公 司, 无水乙醇等购自国药集团化学试剂有限公司, 上述所有试剂均为分析纯, 且未经纯化直接使用。

有机凝胶及其杂化薄膜的微观结构采用 S4800 扫描电子显微镜 (日本 Hitachi)进行观察, 加 速电压为 $15 \mathrm{kV}$, 电流为 $100 \mu \mathrm{A}$, 测试前的薄膜 要经导电处理。有机凝胶样品制备时首先将 0.008 
$\mathrm{g}$ 胶凝剂和 $1 \mathrm{~mL}$ 环己烷加入到特制密封试管中, 加热直至胶凝剂完全溶解, 室温下静置冷却成为 凝胶, 然后将其在液氮中快速冷冻, 经冷冻干燥得 到干凝胶, 再进行上述 SEM 测试。

薄膜的紫外-可见光谱采用 UV-3600 紫外-可 见光分光光度计(日本岛津公司)测试。薄膜的 $X$ 射 线衍射分析图谱采用 $\mathrm{D} / \mathrm{Max}-3 \mathrm{C}$ (日本理学)全自动 $X$ 射线衍射仪测试, 加速电压为 $35 \mathrm{kV}$, 管电流为 $40 \mathrm{~mA}$, 扫描速度为 $0.02\left(^{\circ}\right) \cdot \mathrm{s}^{-1}, 2 \theta$ 范围为 $10^{\circ}-$ $80^{\circ}$ 。

$\mathrm{ZnS}$ 纳米粒子的透射电镜照片采用 JEM$2100 \mathrm{~F}$ 透射电子显微镜(日本电子公司)进行表征。 将 $\mathrm{ZnS}$ 粒子分散于乙醇溶液中, 超声分散 $30 \mathrm{~min}$ 即可。制备 TEM 元素分布图试样时, 首先用刀片 将样品从玻璃基质表面平刮于样品管中, 然后将 其分散于乙醇溶液中, 超声分散 $30 \mathrm{~min}$, 吸取上 述清液, 滴于碳膜铜网表面, 测试电压控制为 200 $\mathrm{kV}$ 。

采用 LS 55 荧光/磷光/发光光谱仪(美国 Perkin Elmer)测试杂化薄膜的发光光谱。猝灭测试的方法 如下: 首先将苂光薄膜固定于比色血中, 并向其中 小心加入 $10 \mu \mathrm{L}$ 待测溶剂, 迅速加盖并用封口膜 密封, 每隔 $2 \mathrm{~min}$ 记录一次薄膜的发射光谱。猝灭 效率采用方程 $\left(I_{0}-I\right) / I_{0} \times 100 \%$ 来计算。式中, $I$ 和 $I_{0}$ 分别为猝灭平衡和在空气中杂化薄膜的苂光发 射强度。

\section{2 实验过程}

\subsection{1 油水界面法制备 ZnS 纳米粒子}

称取 $1.3 \mathrm{~g}$ 硬脂酸锌置于 $250 \mathrm{~mL}$ 四口烧瓶中, 通入氮气 $20 \mathrm{~min}$, 再加入溶有油酸的环己烷溶液 $(4.7 \%(w$, 质量分数 $))$, 磁力搅拌, 加热至 $80^{\circ} \mathrm{C}$, 得淡黄色透明溶液。配制浓度为 $0.16 \mathrm{~mol} \cdot \mathrm{L}^{-1}$ 的 $\mathrm{Na}_{2} \mathrm{~S}$ 水溶液 $25 \mathrm{~mL}$, 并将其利用恒压漏斗缓慢滴 加于上述硬脂酸锌的环已烷溶液中, 电动搅拌上 层油相, 下层水相采用磁力搅拌, $75^{\circ} \mathrm{C}$ 下反应 2 $\mathrm{h}$, 静置 $20 \mathrm{~min}$ 后, 分离上层油相加入热无水乙醇 静置 $30 \mathrm{~min}$, 再离心洗涤 4-5 次, $70^{\circ} \mathrm{C}$ 条件下真 空干燥 $12 \mathrm{~h}$ 后得到 $\mathrm{ZnS}$ 纳米粉体。

\subsubsection{ZnS-超分子有机凝胶杂化薄膜的制备}

参照文献 23 所报道的方法合成胶凝剂 L-丙氨 酸胆固醇酯丁二酸双酰胺, 结构式见图 1, 以环己 烷作为有机胶凝溶剂, 分别称取不同质量的 $\mathrm{ZnS}$ 纳米粒子 $(\mathrm{ZnS}$-有机凝胶杂化薄膜中 $\mathrm{ZnS}$ 含量分别 为 $0.3 \% 、 3.0 \% 、 30.0 \%(w))$ 超声分散于环己烷溶 剂, 得到不同质量浓度的 $\mathrm{ZnS}$ 纳米分散液。

将 $0.008 \mathrm{~g}$ 胶凝剂和 $1 \mathrm{~mL}$ 上述纳米分散液缓

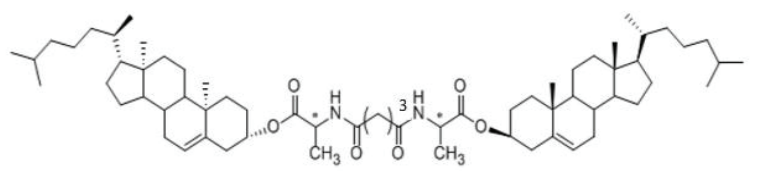

图 1 小分子胶凝剂结构式

Fig. 1 The molecular structure of the LMOG.

慢加入到密封特制试管中, 加热直至胶凝剂彻底 溶解。然后吸取约 $200 \mu \mathrm{L}$ 热溶液流延于预先处理 干净且预热的玻璃基片表面 $(1.4 \mathrm{~cm} \times 3.8 \mathrm{~cm})$, 将 其置于室温下自然干燥, 最后得到稳定的掺杂 $\mathrm{ZnS}$ 的有机凝胶薄膜。

\subsubsection{ZnS-超分子有机凝胶杂化薄膜的传感可} 逆性测试

测试过程如下: (1) 将苂光薄膜固定在比色典 中, 盖上密封盖, 测定在空气中杂化薄膜的荧光发 射强度; (2) 在比色血中小心加入 $10 \mu \mathrm{L}$ 乙二胺后 立即密封比色典; (3) $14 \mathrm{~min}$ 后再次记录苂光发射 强度; (4) 每次测试完毕, 利用电吹风在室温下将 薄膜吹 $10 \mathrm{~min}$ 。该过程至少重复 3 次。

\section{3 结果与讨论}

\section{1 薄膜结构表征}

图 $2 \mathrm{a}$ 为合成的 $\mathrm{ZnS}$ 纳米粒子分散于环己烷溶 液中的 TEM 图片和 $\mathrm{ZnS}$ 粉末的 SEM 图片(图 2b)。 从图 2 可以看出, $\mathrm{ZnS}$ 纳米粒子为球形, 平均粒 径约为 $200 \mathrm{~nm}$, 并且颗粒大小均匀。从图 $2 \mathrm{~b}$ 发现 $\mathrm{ZnS}$ 纳米微粒发生了团聚, 平均粒径增大, 有些颗
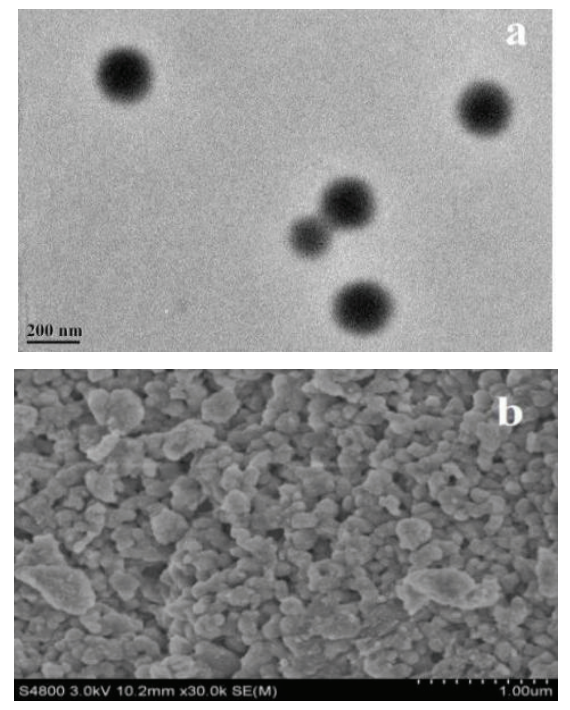

图 2 分散于环己烷溶剂中的 $\mathrm{ZnS}$ 纳米粒子的 TEM 照片(a)和 $\mathrm{ZnS}$ 纳米粒子的 SEM 照片(b)

Fig. 2 The TEM image of $\mathrm{ZnS}$ nanoparticles dispersed in cyclohexane (a) and SEM image of ZnS nanoparticles (b). 
粒的粒径甚至高达 400-600 nm, 推测可能是由于 纳米粒子的粒径较小, 比表面积较大, 表面能较高 导致团聚现象较为明显, 粒径增大。

通过 XRD 手段对杂化薄膜中 $\mathrm{ZnS}$ 的晶型结 构进行了分析, 图 3 为杂化薄膜的 XRD 图谱。可 以看出样品的特征衍射峰出现在 $2 \theta$ 为 $28.5^{\circ}, 47.6^{\circ}$ 和 $56.5^{\circ}$ 三处, 这与立方晶相 $\mathrm{ZnS}$ 的(111), (220), (311)三个点阵面的衍射峰一致[JCPDS 19-0191]。 表明采用油水界面法制备的 $\mathrm{ZnS}$ 纳米粒子为立方 晶型 24 。

图 4 为 $\mathrm{ZnS}$ 担载量不同的杂化薄膜的静态苂 光发射光谱。激发波长均为 $400 \mathrm{~nm}$ 。由图可以看 出, 随着杂化薄膜中 $\mathrm{ZnS}$ 担载量增大, 即从 0 增 大到 $30.0 \%$, 杂化薄膜的静态荧光强度逐渐增强, 发射光谱的形状以及其最大发射峰的位置几乎没 有发生变化, 表明纳米 $\mathrm{ZnS}$ 在不同杂化薄膜中有 效发光粒子粒径基本相同, 这与前面的 $\mathrm{ZnS}$ 纳米 粒子的 TEM 结果基本一致, 其颗粒大小较为均 匀。另外, 随着杂化薄膜中 $\mathrm{ZnS}$ 担载量的增大, 发 光纳米粒子的数量逐渐增多, 故杂化薄膜苂光发 射强度也随之增大。

图 5 为 $\mathrm{ZnS}$ 不同担载量的杂化薄膜的紫外-可 见光谱。可以看出, 杂化薄膜的紫外吸收边基本没 有变化, 均处于 $290 \mathrm{~nm}$ 附近, 与 $\mathrm{ZnS}$ 体材料(禁 带宽 $3.6 \mathrm{eV}$ )的吸收波长 $344 \mathrm{~nm}$ 相比 ${ }^{25}$, 蓝移了 $54 \mathrm{~nm}$, 这是由于纳米材料具有突出的表面效应和 量子尺寸效应, 故表现出明显的吸收带蓝移现象, 这也是纳米材料普遍存在的光学特性之一 25 。进 一步观察发现随着 $\mathrm{ZnS}$ 担载量的增大, 吸收强度 逐渐增大, 与图 4 得到的结论一致。

值得说明的是, 单独 $\mathrm{ZnS}$ 纳米粒子在空气中 存放半个月后, 由于团聚使得 $\mathrm{ZnS}$ 粒子粒径变大

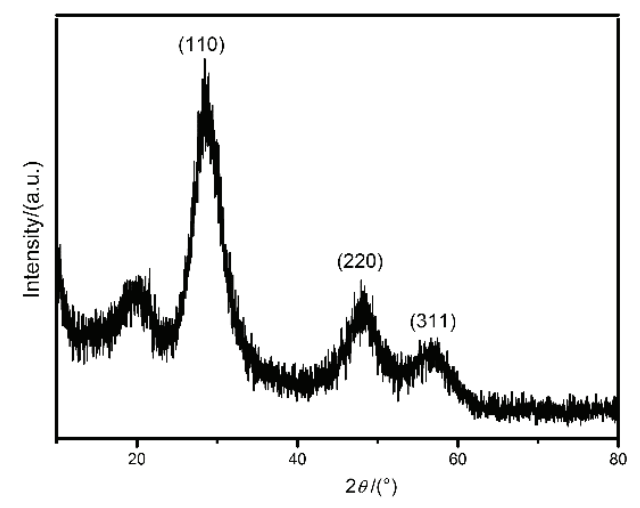

图 $3 \mathrm{ZnS}$-有机凝胶杂化薄膜的 XRD 图谱

Fig. 3 The XRD patterns of the ZnS-organogels hybrid film.

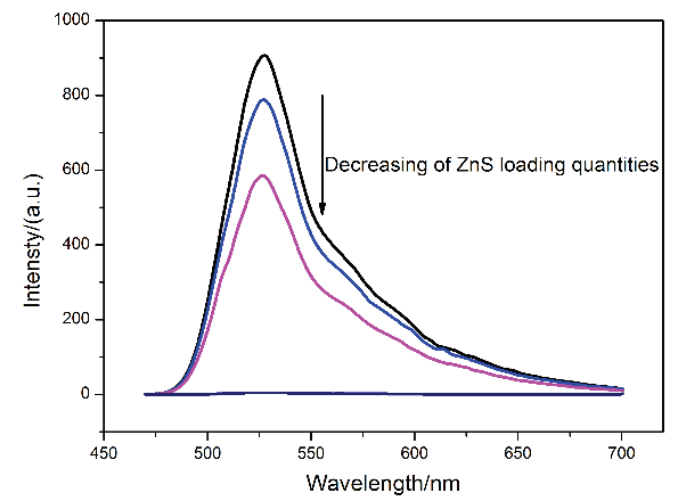

图 $4 \mathrm{ZnS}$ 担载量不同的杂化薄膜 $(30.0 \%, 3.0 \%, 0.3 \%$ and $0 \%(w))$ 的静态荧光发射光谱 $\left(\lambda_{\mathrm{ex}}=400 \mathrm{~nm}\right)$

Fig. 4 The Photoluminescence spectra of the $\mathrm{ZnS}$ organogel hybrid films containing $30.0 \%, 3.0 \%$, $0.3 \%$ and $0 \%(w)$ of $\mathrm{ZnS}\left(\lambda_{\mathrm{ex}}=400 \mathrm{~nm}\right)$.

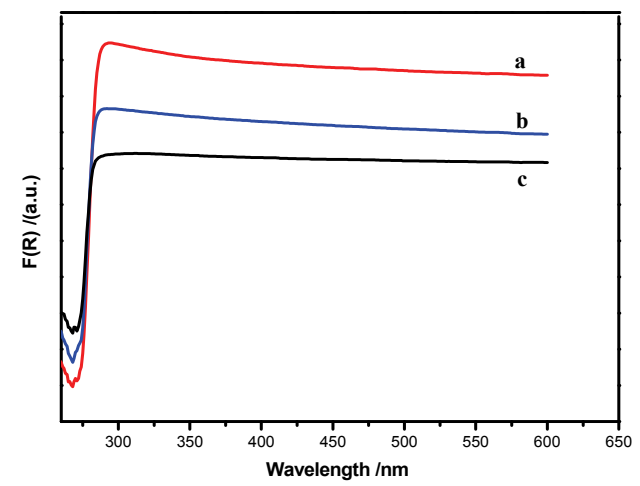

图 $5 \mathrm{ZnS}$ 担载量为 $30.0 \%(\mathrm{a}), 3.0 \%(\mathrm{~b})$ 和 $0.3 \%(w)(c)$ 的 $\mathrm{ZnS}$ 有机凝胶膜的紫外-可见光谱

Fig. $5 \mathrm{UV}$-Vis spectra of the $\mathrm{ZnS}$-organogel films with the loading amount of $30.0 \%$ (a), $3.0 \%$ (b) and $0.3 \%$ (w) (c) $\mathrm{ZnS}$.

导致荧光光谱发生红移, 如图 6a 所示; 相比之下, 杂化薄膜在干燥器中保存 3 个月其荧光光谱最大 发射波长及强度均无明显变化, 表明超分子凝胶 中的三维网络结构可以有效限域和稳定 $\mathrm{ZnS}$ 纳米 粒子(见图 6b), 阻止其聚集, 进一步表明超分子凝 胶是无机纳米粒子的良好载体。

为了考察 $\mathrm{ZnS}$ 纳米粒子在杂化薄膜中的分布 情况, 采用 TEM-EDS 元素分布图像对样品进行了 表征, 图 7a 为杂化薄膜的 TEM 照片, 图 7b, c 分 别为 $7 \mathrm{a}$ 样品中硫元素和锌元素分布图, 可看出两 种元素成功担载于薄膜基质中, 且在薄膜中分布 均一。

\section{2 传感行为}

3.2.1 ZnS 担载量不同的杂化薄膜对 EDA 蒸汽 的传感行为 

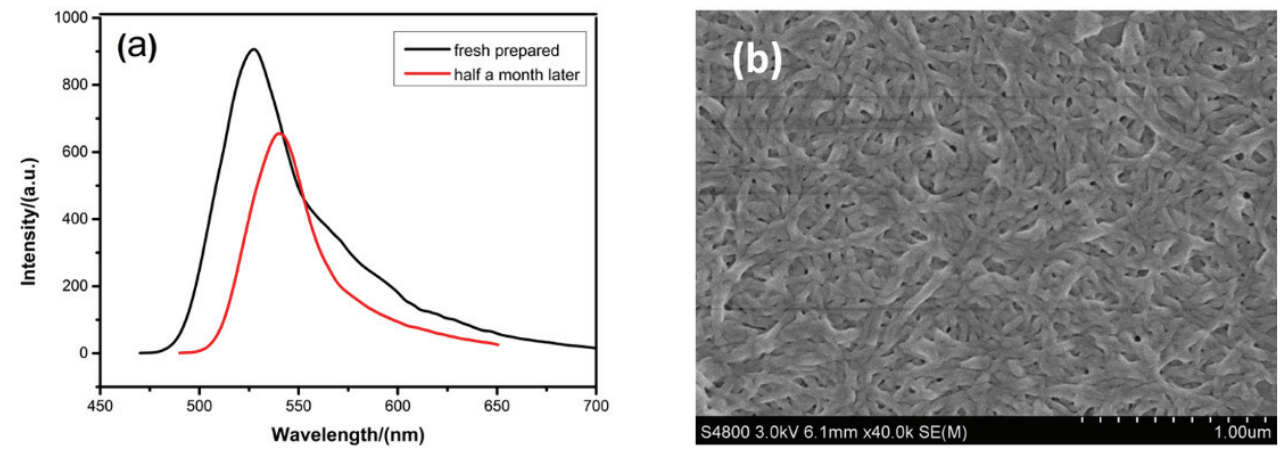

图 $6 \mathrm{ZnS}$ 纳米粒子存放半个月后的 PL 图谱(a)和超分子有机凝胶的 SEM 图片(b)

Fig. 6 PL spectra of ZnS nanoparticles stored for half a month (a) and SEM images of organic gels (b).
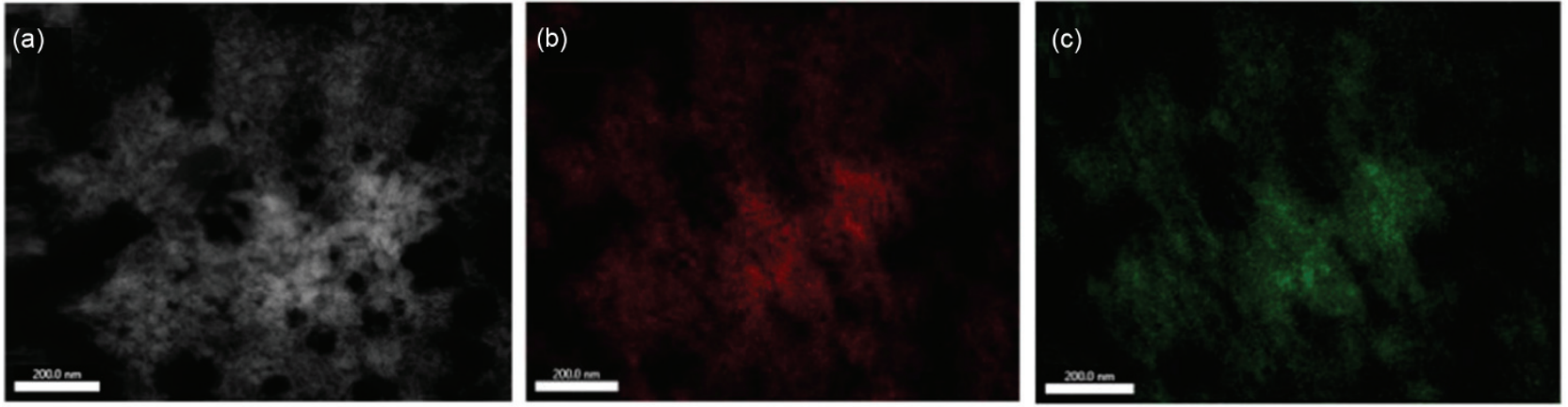

图 7 杂化薄膜的 TEM 照片(a), 图(a)样品中硫元素(b)和锌元素(c)的 TEM-EDS 元素分布图

Fig. 7 TEM image of a fragment of hybrid film (a) and TEM-EDS elemental mapping images of S (b) and $\mathrm{Zn}$ (c) in the hybrid film of the figure (a).
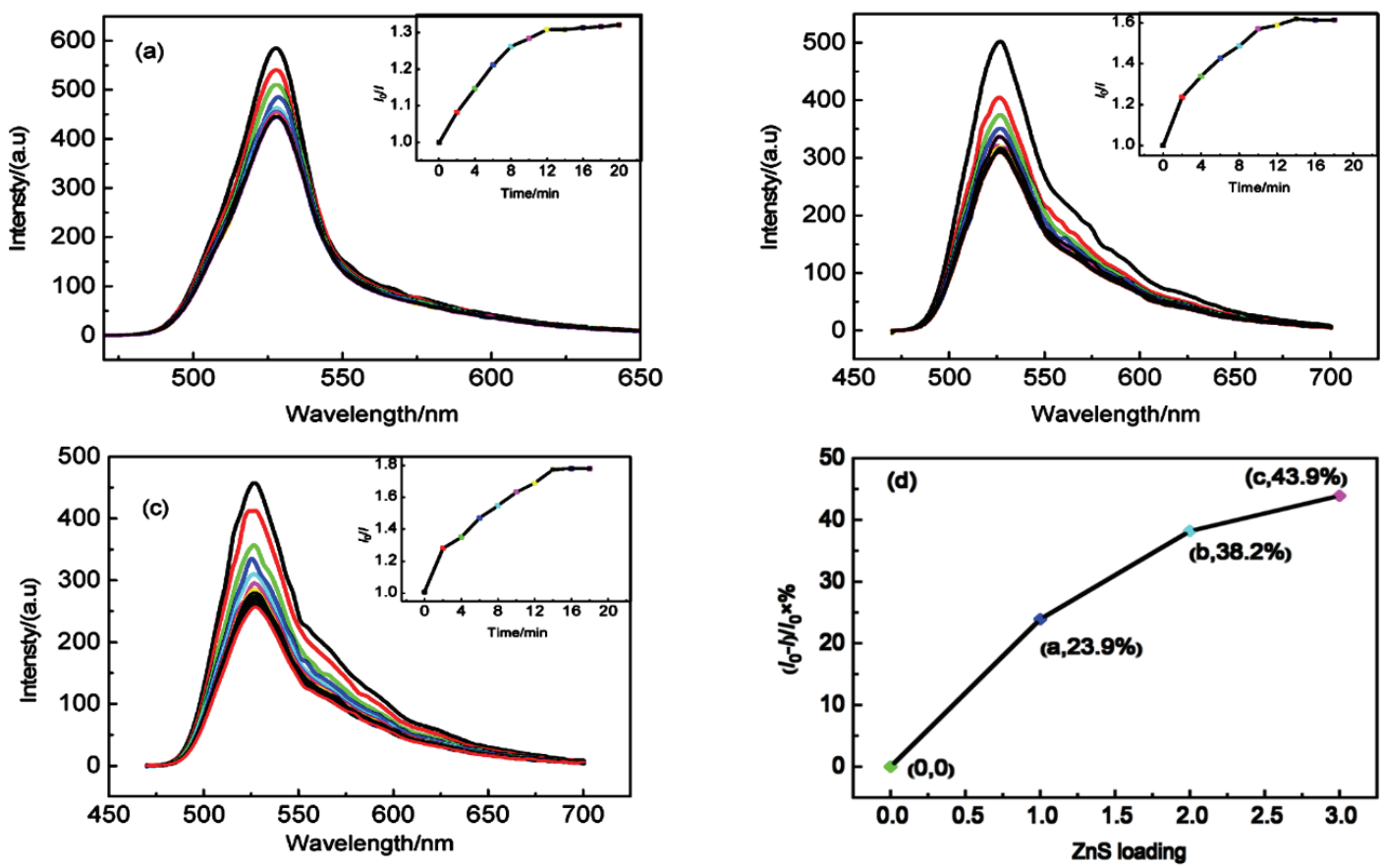

图 $8 \mathrm{ZnS}$ 担载量为 $0.3 \%(\mathrm{a}), 3.0 \%(\mathrm{~b})$ 和 $30.0 \%(w)(\mathrm{c})$ 的 $\mathrm{ZnS}$-有机凝胶杂化膜的最大猝灭效率 $\left(\lambda_{\mathrm{ex}}=400 \mathrm{~nm}\right)$

Fig. 8 The maximum quenching efficiencies of the ZnS-organogel hybrid films containing $0.3 \%$ (a), 3.0\% (b) and $30 \%(w)(c)$ of $\mathrm{ZnS}$, respectively $\left(\lambda_{\mathrm{ex}}=400 \mathrm{~nm}\right)$.

图 $8 \mathrm{a}-\mathrm{c}$ 为 $\mathrm{ZnS}$ 担载量分别为 $0.3 \%$ (a)、3.0\% (b)和 $30.0 \%(w)(\mathrm{c})$ 的杂化薄膜对为相同浓度 EDA
蒸汽的传感情况, 每隔 2 min 扫描一次发射光谱, 可以发现 EDA 蒸汽对三种薄膜的苂光发射都有明 
显的猝灭效果, 且均随着时间的延长, 猝灭效率逐 渐增大, 最后猝灭达到平衡。图 $8 \mathrm{~d}$ 为三种薄膜对 $\mathrm{EDA}$ 蒸汽的最大猝灭效率, 由图可以看出, 随着 $\mathrm{ZnS}$ 担载量的增大, 杂化薄膜对 EDA 的最大猝灭 效率分别为 $23.9 \% 、 38.2 \%$ 和 $43.9 \%$, 灵敏度逐 渐提高; 进一步观察还发现随着 $\mathrm{ZnS}$ 担载量增大, 薄膜的荧光发射对 EDA 蒸汽的响应时间逐渐缩 短。结合之前的研究结果, 推测杂化薄膜对 EDA 的猝灭主要是由于 EDA 与 $\mathrm{Zn}^{2+}$ 发生了络合作用, 即 EDA 蒸汽浓度相同的条件下, 杂化薄膜中 $\mathrm{ZnS}$ 担载量越高, 能够与 $\mathrm{EDA}$ 发生络合作用的 $\mathrm{Zn}^{2+}$ 越 多, 生成络合物的速率越快, 最终生成的络合物越 多, 故猝灭效率逐渐增大, 且响应时间缩短。采用 文献报道 ${ }^{26}$ 方法可计算出 $\mathrm{ZnS}$ 担载量 $30.0 \%$ 时, 检出限为 $10.13 \mathrm{ppm}\left(1 \mathrm{ppm}=1 \times 10^{-6}\right.$ (体积分数) $)$ 。

\subsection{2 选择性}

选择性是衡量苂光传感薄膜的重要指标之 一。故本文将常见挥发性试剂对该薄膜荧光发射 的影响进行了系统研究, 如图 9 所示。由图可知, 在所考察的有机多胺中, 挥发性有机单胺和二胺, 如乙二胺、丙二胺、丁二胺、苯胺对杂化薄膜有较 强的猝灭效率; 相比之下, 氨水几乎不会影响杂化 薄膜的苂光发射; 但甲胺和正丁胺表现出一定的 猝灭效应; 仲胺和叔胺对荧光发射仅有轻微的猝 灭效应, 如, 三乙胺、 $N, N$-二甲基苯胺和二乙胺对 杂化薄膜的最大猝灭效率为 $10 \% 、 8 \%$ 和 $8 \%$; 醇 类试剂表现出轻微的猝灭效应, 如, 甲醇和乙醇的 最大猝灭效率都为 $2 \%$ 左右; 其它挥发性溶剂, 如 丙酮、醋酸和水的最大猝灭效率都低于 $4 \%$; 然而, 固体胺如十二胺、乙酰胺等由于挥发性较弱对该 杂化薄膜的荧光发射几乎没有影响。

\section{3 苂光寿命及猝灭机理}

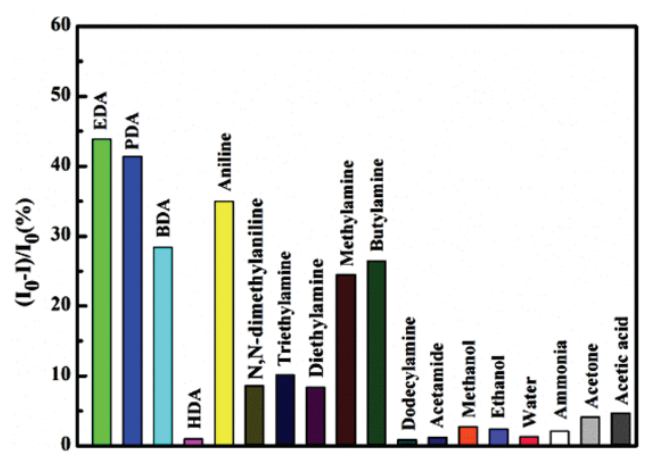

图 9 常用有机溶剂对杂化薄膜荧光发射的最大猝灭效率

$(\mathrm{ZnS}, 30.0 \%)\left(\lambda_{\mathrm{ex}}=400 \mathrm{~nm}\right)$

Fig. 9 The maximum quenching efficiencies of commonly organic solvents on the PL emission of hybrid films

$(\mathrm{ZnS}, 30.0 \%)\left(\lambda_{\mathrm{ex}}=400 \mathrm{~nm}\right)$.
苂光猝灭按照猝灭机理可以分为静态猝灭和 动态猝灭两类。静态猝灭的产生是由荧光物种和 猝灭剂分子之间发生杂化生成一种不发光的杂化 物所致, 静态猝灭并不影响荧光物种的寿命; 动态 猝灭是由一个扩散控制的激发态荧光物种与猝灭 剂相互碰撞的过程所致。其中， $\tau_{0} / \tau=I_{0} / I ， \tau_{0}$ 和 $\tau$ 分别代表发光薄膜在空气中或猝灭剂存在下的平 均荧光寿命 26,27 。因此, 可以通过比较不同猝灭剂 浓度下薄膜的苂光强度与苂光寿命的大小来揭示 猝灭本质。

图 10 为杂化薄膜暴露于 EDA 蒸汽中其苂光 强度随时间的变化曲线。插图为猝灭过程中 $I_{0} / I$ 随 时间的变化曲线。显然, 在前 $10 \mathrm{~min}$ 内, 苂光强 度降低较快, 进一步发现, 整个猝灭过程可以分为 两个阶段, 即第一阶段的快猝灭过程和第二阶段 的慢猝灭过程, 表明传感薄膜中一些光发射中心 处于相对暴露的位置, 容易接触猝灭气体, 表现为 快猝灭, 而其它发射中心可能相对比较隐蔽, 与猝 灭气体较难接触, 导致慢猝灭。

图 11 为 $\mathrm{ZnS}$-有机凝胶发光薄膜分别在空气 和乙二胺蒸汽中达到平衡时的苂光衰减曲线。由 图可知, 在猝灭达到平衡时, $\tau_{0} / \tau=1.08$, 远小于 $I_{0} / I$ (1.6), 表明 EDA 对荧光薄膜的猝灭以静态猝 灭为主 ${ }^{27}, \mathrm{EDA}$ 分子将静态结合于薄膜中 $\mathrm{ZnS}$ 纳 米粒子的缺陷部位, 进而阻止这些部位参与辐射 杂化过程, 因此猝灭了苂光发射 28,29 。Eills 及其合 作者 ${ }^{30}$ 也曾报道单胺和二胺类化合物对 $\mathrm{Zn}^{2+}$ 都有 较强的络合作用。另外, 较小粒径 $\mathrm{ZnS}$ 纳米粒子 往往表现出动态猝灭 ${ }^{28}$, 是本工作中少数动态猝 灭的来源。

\section{4 传感可逆性}

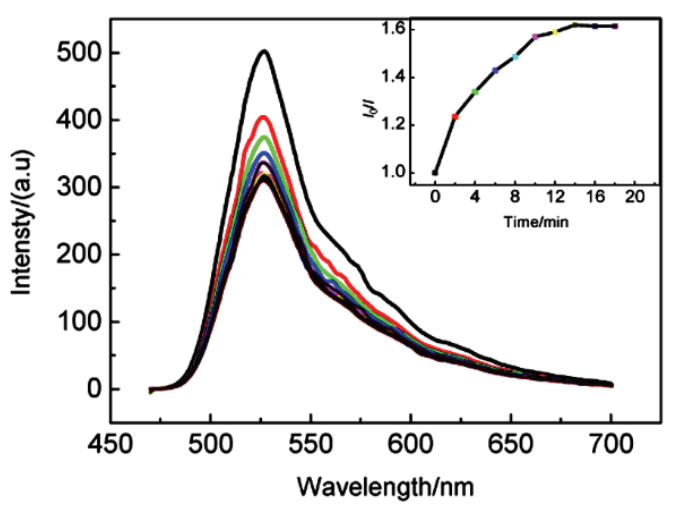

图 10 暴露于 EDA 蒸汽中杂化薄膜的苂光强度随时间 变化曲线

Fig. 10 The PL emission spectra of hybrid films in EDA vapor at different times.

The inset is the plot of $I_{0} / I$ against the exposure time $\left(\lambda_{\mathrm{ex}}=400 \mathrm{~nm}\right)$. 


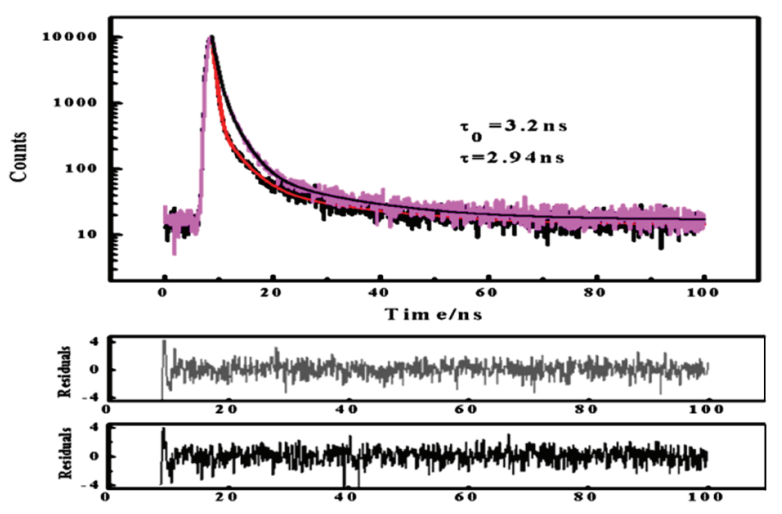

图 $11 \mathrm{ZnS}$-有机凝胶杂化膜在空气(upper)和 EDA 蒸汽中 达到猝灭平衡时 (bottom) 的荧光衰减曲线 $\left(\lambda_{\mathrm{ex}}=400 \mathrm{~nm}\right.$ )

Fig. 11 The PL emission decay profiles of the hybrid film, the upper one measured in the absence of EDA vapor, the bottom one taken in the presence of EDA vapor at the equilibrium state $\left(\lambda_{\mathrm{ex}}=\mathbf{4 0 0} \mathrm{nm}\right)$.

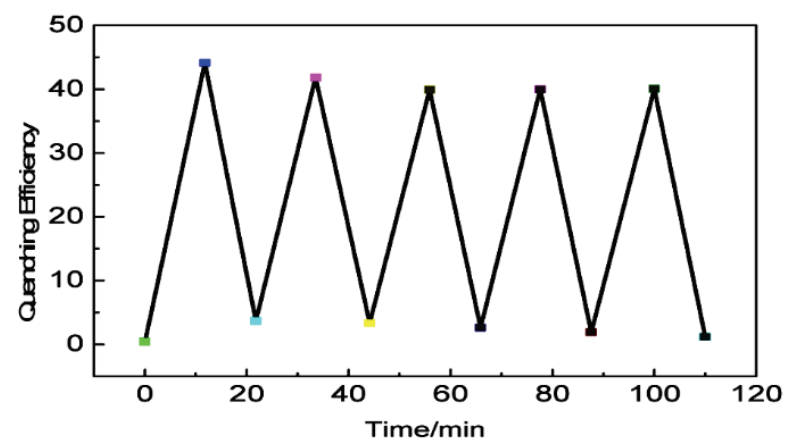

图 $12 \mathrm{ZnS}$-有机凝胶杂化膜对 EDA 响应的可逆性 $\left(\lambda_{\mathrm{ex}}=400 \mathrm{~nm}\right)$

Fig. 12 The reversibility of the response of ZnS-organogel hybrid films to EDA $\left(\lambda_{\mathrm{ex}}=400 \mathrm{~nm}\right)$.

可逆性是传感薄膜的另外一个重要检测指 标。按照实验部分 2.2.3 节进行测试, 重复五次, 结果如图 12 所示。可看出, 在最初两次的测试中 猝灭效率稍有所降低, 但在之后的测试中逐渐趋 于趋于稳定, 恢复性佳, 表明该杂化薄膜对 EDA 蒸汽表现出良好的可逆性传感。

\section{4 结论}

首先采用油水界面法制备 $\mathrm{ZnS}$ 纳米粒子, 制 备的发光 $\mathrm{ZnS}$ 纳米粒子平均粒径为 $200 \mathrm{~nm}$, 再将 其分散于超分子有机凝胶体系中, 通过流延法制 备得到 $\mathrm{ZnS}$ 苂光薄膜。薄膜中的胶凝剂形成的三 维网络结构对 $\mathrm{ZnS}$ 纳米粒子具有良好的限域稳定 作用, 表现为良好的分散性。该发光薄膜能够检测 具有强挥发性的有机单胺及二胺类蒸汽, 灵敏度 高, 选择性好, 并且检测灵敏度随 $\mathrm{ZnS}$ 担载量的
增加而增大, 其猝灭机理以静态猝灭为主。当 $\mathrm{ZnS}$ 担载量 $30.0 \%$ 时, 薄膜对乙二胺蒸汽的检出限为 $10 \mathrm{ppm}$, 可逆性实验表明该薄膜对乙二胺蒸汽具 有良好的可逆响应性

\section{References}

(1) Gao, T.; Tillman, E. S.; Lewis N. S. Chem. Mater. 2005, 17, 2904. doi: $10.1021 / \mathrm{cm} 0494570$

(2) Wei, Q.; Parzuchowski, P.; Zhang, W.; Meyerhoff, M. E. Anal. Chem. 2003, 75, 332. doi: 10.1021/ac0205356

(3) Reviriego, F.; Navarro, P.; Garcia-Espana, E.; Albelda, M. T.; Frias, J. C.; Domenech, A.; Yunta, M. J. R.; Costa, R.; Orti, E. Org. Lett. 2008, 10, 5099. doi: 10.1021/ol801732t

(4) Che, Y. K.; Yang, X. M.; Loser, S.; Zang, L. Nano Lett. 2008, 8, 2219. doi: $10.1021 / \mathrm{n} 1080761 \mathrm{~g}$

(5) Nohta, H.; Satozono, H.; Koiso, K.; Yoshida, H.; Ishida, J.; Yamaguchi, M. Anal. Chem. 2000, 72, 4199. doi: 10.1021/ac0002588

(6) Oberg, K. I.; Hodyss, R.; Beaucham, J. L. Sens. Actuators B 2006, 115, 79. doi: 10.1016/j.snb.2005.08.019

(7) Jin, Y.; Jang, J. W.; Lee, M. H.; Han, C. H. Clin. Chim. Acta 2006, 364, 260. doi: $10.1021 /$ jf050484o

(8) Xia, H. Y.; He, G.; Peng, J. X.; Li, W. W.; Fang, Y. Appl. Surf. Sci. 2010, 256, 7270. doi: 10.1016/j.apsusc.2010.05.063

(9) Xia, H. Y.; He, G.; Gao, L. N.; Peng, J. X.; Fang, Y. Chem. J. Chin. Univ. 2010, 31, 1614. [夏慧芸, 何刚, 高莉宁, 彭军霞, 房喻. 高等学校化学学报, 2010, 31, 1614.] doi: 10.1021/ja0269082

(10) Wang, L.; Lu, D. F; Cheng, J.; Zhang, J., Qi, Z. M. Acta Phys. -Chim. Sin. 2017, 33, 1223. [王丽，逯丹凤，高然，程进，张吉，祁志美. 物理化学学报, 2017, 33, 1223.] doi: 10.3866/ PKU.WHXB201702282

(11) Xia, H. Y.; Liu, G. Y.; Zhao, C.; Meng, X. J.; Li, F. F.; Wang, F. Y.; Duan, L.; Chen, H. X. RSC Adv. 2017, 7, 17264. doi: 10.1039/C7RA00556C

(12) Wu, H. F.; Chen, Y.; Xu, S. H.; Yan, Y. H.; Si, J. X.; Tan, Y. S. Acta Phys. -Chim. Sin. 2017, 33, 419. [ 吴海飞, 陈耀, 徐珊瑚, 鄢永红, 斯剑霄, 谭永胜. 物理化学学报, 2017, 33, 419.] doi: 10.3866/PKU.WHXB201610192

(13) Murata, K.; Aoki, M.; Suzuki, T.; Harada, T.; Kawabata, H.; Komori, T.; Ohseto, F.; Ueda, K.; Shinkai, S. J. Am. Chem. Soc. 1994, 116, 6664. doi: 10.1021/ja00094a023

(14) Shinkai, S.; Murata, K. J. Mater. Chem. 1998, 8, 485. doi: $10.1039 / \mathrm{A} 704820 \mathrm{C}$

(15) Yoza, K.; Amanokura, N.; Ono, Y. Chem. Eur. J. 1999, 5, 2722. doi: 10.1002/(SICI)1521-3765(19990903)5:9<2722::AIDCHEM2722>3.0.CO; $2-\mathrm{N}$

(16) George, M.; Weiss, R. G. Acc. Chem. Res. 2006, 39, 489. 
doi: $10.1002 / \operatorname{chin} .200645254$

(17) Jyothish, K.; Hariharan, M.; Ramaiah, D. Chem. Eur. J. 2007, 13, 5944. doi: 10.1002/chem.200700130

(18) Xia, H. Y.; Peng, J. X.; Liu, K. Q.; Li, C.; Fang, Y. J. Phys. D: Appl. Phys. 2008, 41, 105405. doi: 10.1088/0022-3727/41/10/105405

(19) Zou, Z.; Qiu, Y.; Xie, C.; Xu, J.; Luo, Y.; Wang, C.; Yan, H. J. Alloy. Compd. 2015, 645, 17. doi: 10.1016/j.jallcom.2015.04.20

(20) Xu, K.; Li, N.; Zeng, D.; Tian, S.; Zhang, S.; Hu, D.; Xie, C. ACS Appl. Mater. Interfaces 2015, 7, 11359. doi: 10.1021/acsami.5b01856

(21) Gaiardo, A.; Fabbri, B.; Guidi, V.; Bellutti, P.; Giberti, A.; Gherardi, S.; Vanzetti, L.; Malagù, C.; Zonta, G. Sensors 2016, 16, 296. doi: $10.3390 / \mathrm{s} 16030296$

(22) Fu, X.; Liu, J.; Wan, Y.; Zhang, X.; Meng, F.; Liu, J. J. Mater. Chem. 2012, 22, 17782. doi: 10.1039/C1JM14032A

(23) Peng, J. X.; Liu, K. Q.; Liu, J.; Zhang, Q. H.; Feng, X. L.; Fang, Y. Langmuir 2008, 24, 2992. doi: 10.1021/la703672u

(24) Ghosh, G.; Naskar, M. K.; Patra, A. J. Opt. Mater. 2006, 28, 1047. doi: 10.1016/j.optmat.2005.06.003

(25) Lippens, P. E.; Lanoo. M. Phys. Rev. B: Condens. Matter 1989, 39, 10935. doi: 10.1103/PhysRevB.39.10935

(26) Du, H. Y.; He, G.; Liu, T. H.; Ding, L. P.; Fang, Y. J. Photochem. Photobiol. A: Chem. 2010, 217, 356. doi: 10.1016/j.jphotochem. 2010.11.004

(27) Lakowicz, J. R. Time-Resolved Energy Transfer and Conformational Distrbutions of Biopolymers, 3rd ed.; Springer: USA, 1999. pp. 395-424.

(28) Landes, C. F.; Braun, M.; El-Sayed, M. A. J. Phys. Chem. B 2001, 105, 10554. doi: 10.1021/jp0118726

(29) Selmarten, D.; Jones, M.; Rumbles, G.; Yu, P.; Nedeljkovic, J.; Shaheen, S. J. Phys. Chem. B 2005, 109 (33), 15927. doi: $10.1021 /$ jp0515479

(30) Lisensky, G. C.; Penn, R. L.; Murphy, C.; Eills, A. B. Science 1990, 248, 840. doi: 10.1126/science.248.4957.840 